\title{
Brexit - Auswirkungen auf den deutschen und europäischen Luftverkehr
}

\author{
Eva-Maria Cronrath, Sven Maertens, Peter Berster, Marc Gelhausen \\ Deutsches Zentrum für Luft- und Raumfahrt e.V. (DLR), Institut für Flughafenwesen und \\ Luftverkehr \\ Linder Höhe, 51147 Köln
}

Die Autoren sind wissenschaftliche Mitarbeiter am Institut für Flughafenwesen und Luftverkehr des Deutschen Zentrums für Luft- und Raumfahrt e.V. in Köln und forschen schwerpunktmäßig in den Bereichen Luftverkehrsprognosen, auch unter Berücksichtigung von

Kapazitätsrestriktionen, sowie Angebots- und Wettbewerbsanalysen und Markt- und Umweltregulierung des Luftverkehrs.

\section{Abstract englisch: \\ Brexit - Consequences for German and European Air Traffic}

A Brexit would have notable impact on air traffic in Germany and Europe. Approximately a quarter of all inner-European flights is potentially affected. Up to $14 \%$ are likely to become impossible under new post-Brexit legal arrangements. These endangered connections are mainly served by Ryanair and easyJet. In case the two low cost carriers restructured their businesses, the European market's supply structure could change distinctly. On the demand side (regardless of supply side and exchange rate effects) the forecasted economic downturn would lead to a decrease in German air traffic growth by $0.1 \%$-points.

\begin{abstract}
deutsch:
Die Auswirkungen eines Brexit auf den Luftverkehr in Deutschland und Europa wären deutlich spürbar. Etwa ein Viertel der innereuropäischen Verbindungen ist potenziell vom Brexit betroffen. Bis zu 14\% gelten aufgrund der Rechtslage als besonders gefährdet wegzufallen. Diese Verbindungen werden vor allem von Ryanair und easyJet angeboten. Sollten beide Billigflieger umstrukturieren, könnte sich die Angebotsstruktur im EU-Luftverkehr deutlich verändern. Auf der Nachfrageseite (ohne Berücksichtigung von Angebots- und Währungseffekten) ist durch sinkende Wirtschaftsleistung für den deutschen Luftverkehr eine Dämpfung des Wachstums um 0,1\%-Punkte zu erwarten.
\end{abstract}

\section{Text:}

Am 23. Juni 2016 fiel im Vereinigten Königreich von Großbritannien und Nordirland in einem Referendum die Entscheidung, die EU zu verlassen. Der Wert des Britischen Pfunds sank nach 
dem Wahltag auf den tiefsten Stand gegenüber dem US Dollar seit 31 Jahren. ${ }^{1}$ Die Konsequenzen eines „Brexits” für den Luftverkehr könnten ebenfalls erheblich sein, nicht nur für das Vereinigte Königreich, sondern etwa auch für Deutschland. Luftverkehr verbindet die heimische Industrie mit ihren Zuliefer- und Absatzmärkten, ist eine Säule des Tourismus und laut Branchenverband IATA verantwortlich für 62,7 Millionen Arbeitsplätze weltweit. ${ }^{2}$ In Deutschland schätzen nach einer Umfrage des ifo Instituts 56\% aller und 74\% der Industrieunternehmen ihre Luftverkehrsanbindung als wichtig oder sehr wichtig für ihre Geschäftstätigkeit ein. ${ }^{3}$ Welche Folgen ein Brexit für den Luftverkehrsmarkt hätte, wird stark von der tatsächlichen Ausgestaltung eines Austritts abhängen, welche gemäß Artikel 50 des EU-Vertrages binnen zwei Jahren nach formeller Austrittserklärung mit der EU auszuhandeln ist.

Verließe das Vereinigte Königreich tatsächlich die EU, würde dort im Äußersten das gesamte EURecht grundsätzlich nicht mehr gelten und müsste durch neue Rechtsvorschriften ersetzt werden. Für den Luftfahrtsektor wird erwartet, dass "sachgerechte", technische Vorschriften der EU vom Vereinigten Königreich zur Gewährung der Kontinuität übernommen würden, hingegen aber Verkehrs- und damit Marktzugangsrechte, die in der bisherigen EU-Mitgliedschaft begründet sind und über die nicht unilateral entschieden werden kann, nach einem Austritt aus der EU grundsätzlich verloren wären. ${ }^{4}$

\section{Marktzugangsmöglichkeiten im europäischen Luftverkehr}

Fluggesellschaften der EU, also bislang auch britische Unternehmen, sowie die EFTA-Mitglieder Norwegen und Island und einige weitere Staaten dürfen beliebige Routen innerhalb großer Teile Europas bedienen und zudem von dort uneingeschränkt in ausgewählte Drittstaaten wie die USA, Kanada oder Marokko fliegen.

Hintergrund ist die zu den vier Grundfreiheiten des Binnenmarktes zählende Dienstleistungsfreiheit. ${ }^{5}$ Dieser Gedanke wurde für den EU-Binnenluftverkehr sektorspezifisch umgesetzt. ${ }^{6}$ Kern des seit 1997 vollständig implementierten „EU Single Aviation Markets” („EU Open Sky") ist ein grundsätzlich freier Zugang aller EU-Fluggesellschaften zu allen Strecken innerhalb der EU bei freier Preisbildung und harmonisierten Rahmenbedingungen in allen Mitgliedsstaaten. Dies bedeutet eine Gewährung aller neun durch die International Civil Aviation Organization (ICAO) definierten Freiheiten der Luft (s. Abbildung 1) und schließt damit selbst Flüge zwischen zwei EU-Drittstaaten (7. Freiheit) und innerhalb eines anderen EU-Staates (9.

\footnotetext{
1 Vgl. Reuters: Sterling pares losses after hitting 31-year low on Brexit, 24.6.2016, http://www.reuters.com/article/us-global-forex-idUSKCN0Z9208 (4.7.2016).

${ }^{2}$ Vgl. International Air Transport Association (IATA): Economic Performance of the Airline Industry, 2016 Mid-year report, o. O. 2016.

${ }^{3} \mathrm{Vgl}$. Bundesverband der deutschen Luftverkehrswirtschaft (BDL) in Kooperation mit ifo Institut: Report Luftfahrt und Wirtschaft, Berlin 2014.

${ }^{4}$ Vgl. E. Giemulla, P. Kortas: Brexit wäre das Ende für einfachen Marktzugang, in: DVZ, Nr. 48, 17.6.2016.

${ }^{5}$ Geregelt in Artikel 56ff. des Vertrags über die Arbeitsweise der EU.

${ }^{6}$ Rechtsverordnungen (EWG) Nr. 2408/92 und (EG) Nr. 1008/2008.
} 
Freiheit, sog. "Kabotage“) ein.7 Die EFTA-Staaten Norwegen und Island sowie ihre Fluggesellschaften sind über die EWR-Mitgliedschaft der beiden Länder eingeschlossen.

Abbildung 1: Freiheiten der Luft

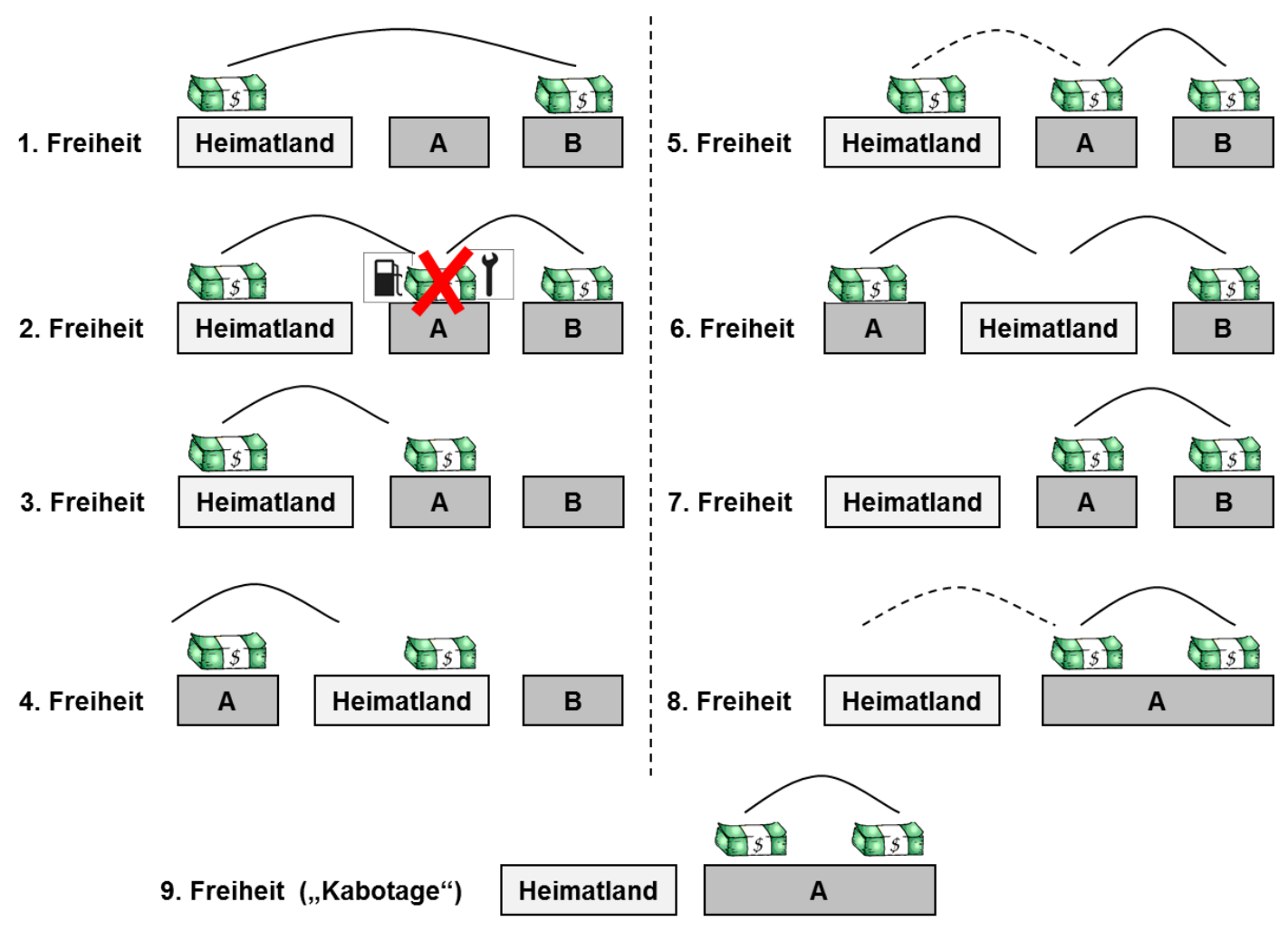

Quelle: Eigene Darstellung basierend auf ICAO Manual on the Regulation of International Air Transport (Doc 9626, Part 4)

In der Praxis bedeutet dies, dass etwa eine irische Fluggesellschaft nicht nur wie früher von und nach Irland (3. und 4. Freiheit), sondern etwa auch zwischen Italien und Spanien (7. Freiheit) oder innerhalb Deutschlands (9. Freiheit) fliegen darf, oder eine britische Fluggesellschaft innerhalb Frankreichs oder von Griechenland in die Niederlande. Folge dieser Marktöffnung war eine insbesondere von dynamisch agierenden Billigfluggesellschaften getriebene Wettbewerbsstimulierung im vorher hauptsächlich durch 3.- und 4.-Freiheitsverkehre staatlicher Monopolisten gekennzeichneten europäischen Luftverkehr. So ist etwa die Zahl der in der EU angebotenen Sommerrouten zwischen 1992 und 2012 um etwa das Anderthalbfache gestiegen. ${ }^{8}$

Eine Erweiterung des liberalisierten europäischen Luftverkehrsmarktes fand durch die Gründung der European Common Aviation Area (ECAA) statt, welche die Westbalkanstaaten Albanien, Bosnien-Herzegowina, Kosovo, Mazedonien, Montenegro und Serbien einschließt, die im Gegenzug die gesetzlichen Rahmenbedingungen des gemeinsamen EU-Luftverkehrsmarktes in Bereichen wie Flug- und Luftsicherheit, Wettbewerb, Beihilfen und Passagierrechte übernahmen

\footnotetext{
${ }^{7}$ Vgl. bspw. B.F. Havel, G.S. Sanchez: The Principles and Practice of International Aviation Law, Cambridge 2014, S. $97 \mathrm{ff}$.

${ }^{8} \mathrm{Vgl}$. European Commission: Fitness Check - Internal Aviation Market, Report on the Suitability of Economic Regulation of the European Air Transport Market and of Selected Ancillary Services, Brüssel, 2013, S. 21 u. 26.
} 
(„regulatory convergence“). Weiterhin zu nennen sind inhaltlich ähnlich ausgerichtete Abkommen mit „Nachbarstaaten“ wie Marokko, Georgien, Jordanien, Moldau und Israel, sowie ebenfalls liberalisierte Abkommen mit "Global Partners" wie den USA oder Kanada, auf die hier angesichts der Beschränkung auf die Folgen eines Brexits für den innereuropäischen Luftverkehr nicht weiter eingegangen wird. ${ }^{9}$

\section{Luftverkehrsrechtliche Bedeutung eines Brexits}

Sollte das Vereinigte Königreich die EU verlassen, wäre es aus Sicht der EU ein Drittstaat. Die beschriebenen Abkommen hätten für das Land dann grundsätzlich keine Gültigkeit mehr, und britische Fluggesellschaften wären keine Gemeinschaftsunternehmen mehr. Dies hieße zum einen, dass diese ihr Recht, Luftverkehr zwischen oder innerhalb anderer Staaten der EU/ECAA durchzuführen, verlieren würden. ${ }^{10}$ Zum anderen würden Fluggesellschaften aus anderen EU/ECAA-Staaten nicht mehr innerhalb des Vereinigten Königreichs bzw. von dort in Drittstaaten fliegen dürfen. Schließlich müsste prinzipiell auch der bilaterale Verkehr von Großbritannien und Nordirland in jeden einzelnen EU/ECAA-Staat neu verhandelt werden. ${ }^{11}$

Es ist jedoch zu erwarten, dass es im bilateralen 3.- und 4.-Freiheitsverkehr zwischen dem Vereinigten Königreich und EU/ECAA aus folgenden Gründen nicht zu einem massiven Einbruch des Verkehrsangebots käme. ${ }^{12}$ Zum einen wird argumentiert, dass alte, bilaterale Abkommen, sofern nicht gekündigt, nach Ende des Anwendungsvorrangs des Europarechts wieder aufleben würden. ${ }^{13}$ Zum anderen ist es angesichts der grundsätzlichen Strategie der EU und ihrer Mitglieder, möglichst liberale Luftverkehrsabkommen mit Drittstaaten auszuhandeln, durchaus wahrscheinlich, dass zumindest ein freier Marktzugang für Fluggesellschaften der unterzeichnenden Staaten in neuen bilateralen Luftverkehrsabkommen ausgehandelt würde. British Airways und easyJet würden also vermutlich weiter unbeschränkt ab London und anderen britischen Städten in die EU operieren können, und eine Lufthansa könnte weiterhin von ihren Drehkreuzen Frankfurt und München britische Ziele anfliegen.

Hingegen ist es ebenfalls wahrscheinlich, dass dem Vereinigten Königreich die Teilnahme am freien Luftverkehrsbinnenmarkt zunächst versagt würde. Dies würde nach Ansicht von Giemulla und Kortas ${ }^{14}$ Flüge von britischen Fluggesellschaften innerhalb oder zwischen EU/ECAA Staaten betreffen, und im Gegenzug auch Flüge von Fluggesellschaften aus EU/ECAA-Staaten innerhalb Großbritanniens oder von anderen EU/ECAA-Staaten dorthin (7. und 9. Freiheit). Die britische easyJet könnte dann also nicht mehr zwischen Frankreich und Italien oder innerhalb Spaniens operieren, und die irische Ryanair dürfte nur noch ab Irland, aber nicht mehr aus anderen EU-

\footnotetext{
${ }^{9}$ Vgl. European Commission: Air - External Aviation Policy, sowie die dort verlinkten Unterseiten http://ec.europa.eu/transport/modes/air/international_aviation/external_aviation_policy/index_en.htm, (4.7.2016).

${ }^{10} \mathrm{Vgl}$. E. Giemulla, P. Kortas, a.a.O.

${ }^{11}$ Vgl. ebd.; vgl. T. Payne, E. Warwick: Brexit: Impact on Aviation Law, DLA Piper, 24.6.2016, https://www.dlapiper.com/de/germany/insights/publications/2016/04/brexit-at-a-glance/brexit-aviation (4.7.2016).

12 Vgl. Frontier Economics: Over and Out - Brexit and Aviation, London 12.7.2016.

${ }^{13}$ Vgl. E. Giemulla, P. Kortas, a.a.O.

${ }^{14} \mathrm{Vgl}$. ebd.
} 
Staaten nach Großbritannien fliegen. Bezüglich der Verkehre der 7. Freiheit von EU-Airlines in ein von der EU losgelöstes Großbritannien erachten wir es jedoch als wahrscheinlich, dass die im Jahr 2002 vom Europäischen Gerichtshof geforderte Außerkraftsetzung sogenannter nationalitätsbedingter Beschränkungen ${ }^{15}$ zumindest zeitnah greifen müsste, wie sie mittlerweile in vielen ( „horizontalen“) Luftverkehrsabkommen zwischen der EU bzw. einzelnen EU-Staaten und Drittstaaten umgesetzt ist. ${ }^{16}$ Airlines wie Ryanair dürften dann doch ab jedem EU-Staat weiter nach Großbritannien fliegen. Dennoch betrachten wir auch diese Relationen aufgrund möglicher Unabwägbarkeiten im Folgenden als gefährdet.

Im Folgenden wird anhand von Flugplandaten dargestellt, welche Bedeutung die als "betroffen" bzw. "gefährdet" identifizierten Luftrechte aus Sicht des Gesamtmarktes und für einzelne Akteure haben.

\section{Ein Viertel der innereuropäischen Flüge potenziell von Brexit betroffen}

Betrachtet man anhand von Flugplandaten des Anbieters OAG (Official Airline Guide) den Flugverkehr allein innerhalb der bisherigen EU28 für das Jahr 2015, wird deutlich, wieviel auf dem Spiel steht. In der folgenden empirischen Betrachtung steht Großbritannien stellvertretend für das Vereinigte Königreich.

Innerhalb der EU28 wurden insgesamt rund 668 Mio. Sitze auf 4,8 Mio. Flügen angeboten (Tabelle 1). Großbritannien und/oder britische Fluggesellschaften betraf davon insgesamt etwa ein Viertel (27\% der Sitze und 25\% der Flüge). Gefährdet sind alle Verkehre der 7. und 9. Freiheit ${ }^{17}$, welche europaweit einen Anteil von 16\% der Sitze und 14\% der Flüge ausmachen.

Tabelle 1: Durch einen Brexit betroffenes und gefährdetes europäisches Flugangebot auf Basis des Jahres 2015 (Datenquelle: OAG Flugpläne)

\begin{tabular}{|c|c|c|c|c|}
\hline von & nach & Fluggesellschaften & Sitze & Flüge \\
\hline EU28 & EU28 & alle & 667.522 .180 & 4.831 .800 \\
\hline EU27 (ohne GB) & GB & alle & 78.245 .054 & 510.503 \\
\hline GB & EU27 (ohne GB) & alle & 78.216 .010 & 511.143 \\
\hline GB & GB & nicht-britische & 2.014 .717 & 17.432 \\
\hline EU27 (ohne GB) & EU27 (ohne GB) & britische & 23.073 .696 & 150.999 \\
\hline \multicolumn{3}{|c|}{$=>$ insgesamt betroffener Luftverkehr } & $(27 \%) 181.549 .477$ & $(25 \%) 1.190 .077$ \\
\hline von & nach & Fluggesellschaften & Sitze & Flüge \\
\hline EU28 & EU28 & alle & 667.522.180 & 4.831 .800 \\
\hline GB & $\begin{array}{l}\text { EU27 (ohne GB), } \\
\text { nicht Heimat }\end{array}$ & $\begin{array}{l}\text { nicht-britische } \\
\text { (7. Freiheit) }\end{array}$ & 39.320 .467 & 251.807 \\
\hline $\begin{array}{l}\text { EU27 (ohne GB), } \\
\text { nicht Heimat }\end{array}$ & GB & $\begin{array}{l}\text { nicht-britische } \\
\text { (7. Freiheit) }\end{array}$ & 39.910 .693 & 255.034 \\
\hline GB & $\mathrm{GB}$ & $\begin{array}{l}\text { nicht-britische } \\
\text { (9. Freiheit) }\end{array}$ & 2.014 .717 & 17.432 \\
\hline EU27 (ohne GB) & EU27 (ohne GB) & $\begin{array}{l}\text { britische } \\
\text { (7.+9. Freiheit) }\end{array}$ & 23.073 .696 & 150.999 \\
\hline
\end{tabular}

${ }^{15}$ Vgl. http://eur-lex.europa.eu/LexUriServ/LexUriServ.do?uri=CELEX:61998C0466:EN:HTML:NOT [Abruf am 4. Juli 2016].

${ }^{16}$ Vgl. M. Schladebach: Lufthoheit: Kontinuität und Wandel, Tübingen, 2014, S.357.

${ }^{17}$ Flüge der 5. und 8. Freiheit sind innerhalb Europas kaum relevant. 
Welche Bedeutung ergibt sich für den deutschen Luftverkehr? Innerhalb Europas starten in Deutschland die meisten Flugzeuge. Im Jahr 2015 wurden von Deutschland aus 92,6 Mio. Sitze in andere EU-Länder geflogen, von Großbritannien aus waren es 41,3 Mio. Die EU war für beide Länder der größte Markt (Abbildung 2 und Abbildung 3). Über die Hälfte der Sitzkapazität auf Flügen aus Großbritannien (51\%) entfiel auf Verbindung in andere EU-Länder, für Deutschland machten Verbindungen in die EU 47\% der Sitzkapazität aus. Die Verflechtung zwischen Deutschland und Großbritannien war dabei besonders eng. Den höchsten Anteil am Flugverkehr aus Großbritannien hatten allerdings Flüge nach Spanien (12\%) und in die USA (8\%). Darauf folgte Deutschland mit 6\%. Andersherum spielte Großbritannien auch für Deutschland eine große Rolle; es stand nach Spanien auf Platz zwei mit einem Verkehrsanteil von ebenfalls $6 \%$. Grund für den starken Verkehrsfluss zwischen Deutschland und dem Vereinigten Königreich ist neben dem privaten Reiseverkehr die enge wirtschaftliche Beziehung der beiden Länder. Das Vereinigte Königreich war für Deutschland in 2015 mit einem Anteil 6\% am gesamten Außenhandelsvolumen der fünftgrößte Handelspartner hinter den USA, Frankreich, den Niederlanden und China. ${ }^{18}$

Abbildung 2: Flugverbindungen von Großbritannien in die Welt (geflogene Sitze in 2015)
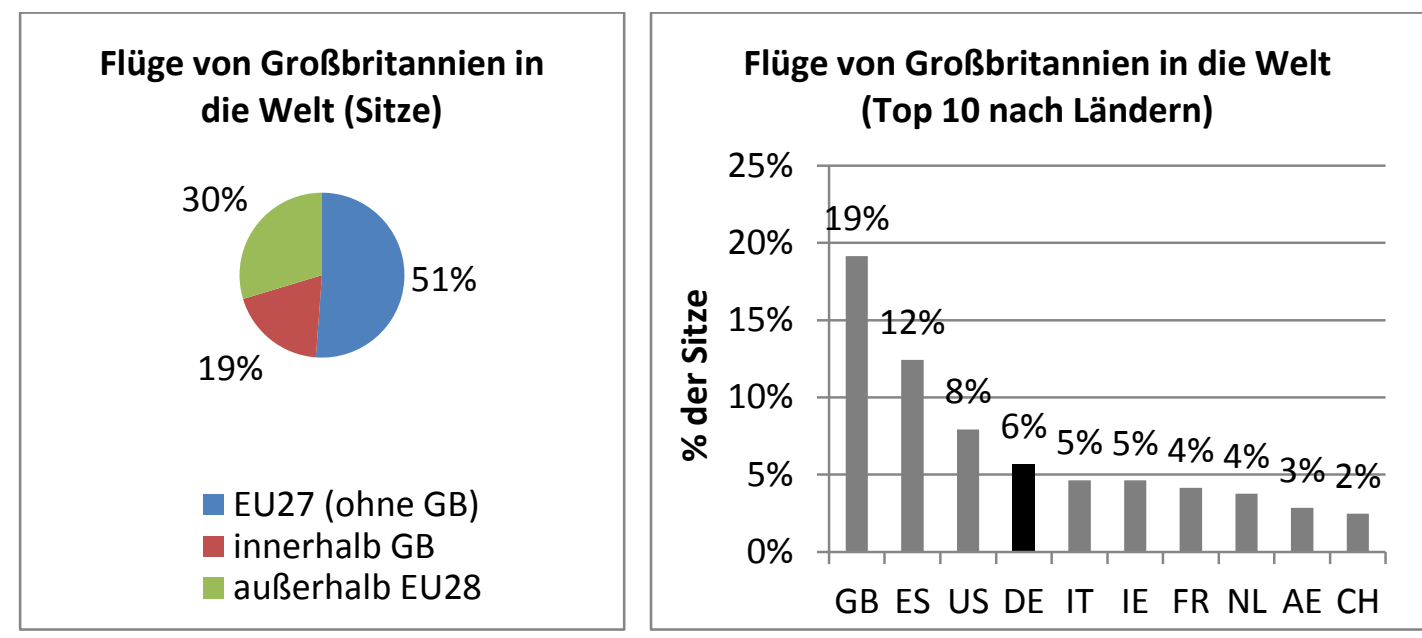

Abbildung 3: Flugverbindungen von Deutschland in die Welt (geflogene Sitze in 2015)

\footnotetext{
${ }^{18}$ Datenquelle: Destatis: Zusammenfassende Übersichten für den Außenhandel (vorläufige Ergebnisse) Jahr 2015, Wiesbaden 3.3.2016.
} 

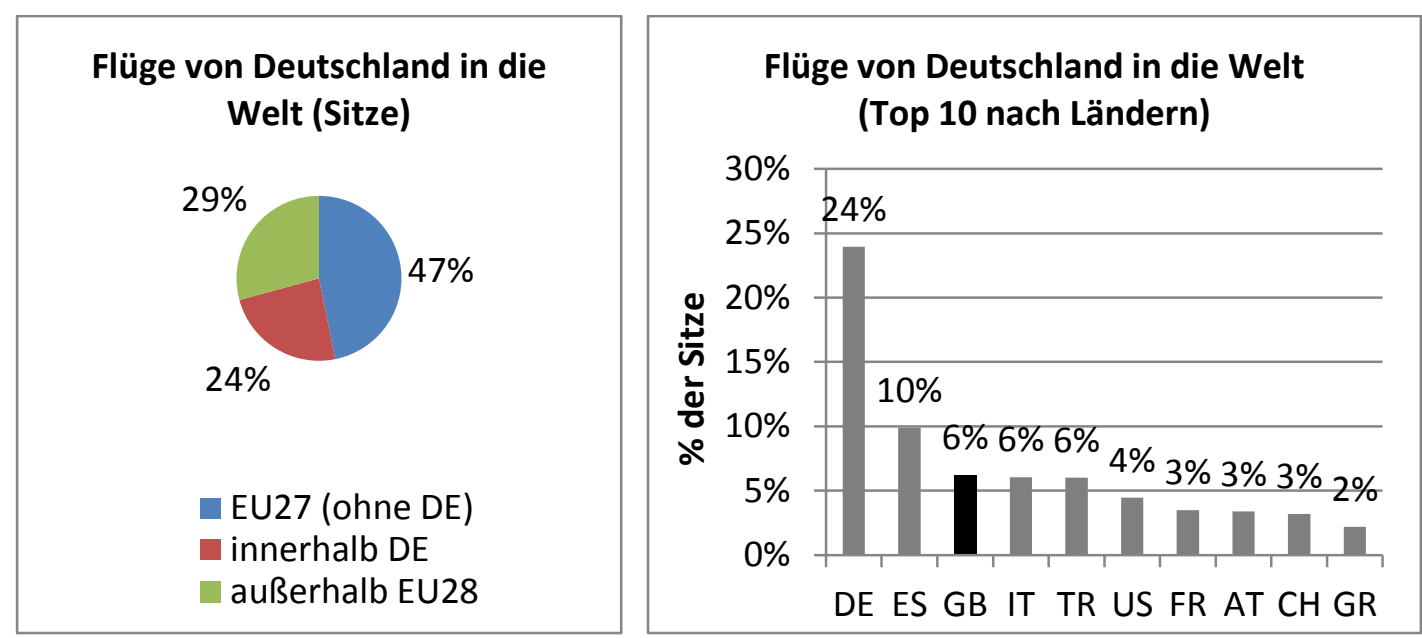

Für den deutschen Luftverkehr wären von einem Brexit etwa 19,4 Mio. Sitze potenziell betroffen (Tabelle 2). Als gefährdet sind die Verbindungen zu bewerten, die 7. und 9. Freiheiten nutzen. Das sind vor allem diejenigen Flüge zwischen Deutschland und Großbritannien, die nicht von britischen oder deutschen Fluggesellschaften durchgeführt werden. Sie machen etwa 15\% der angebotenen Sitzkapazität zwischen den beiden Ländern aus. Innerhalb Großbritanniens sind deutsche Fluggesellschaften kaum tätig, britische bieten innerhalb Deutschlands nahezu keine Verbindungen an. Insgesamt ergibt sich somit für den deutschen Luftverkehr eine Gefährdung von rund 2,7 Mio. Sitzen auf weniger als 15.000 Flügen.

Tabelle 2: Durch einen Brexit betroffenes und gefährdetes deutsch-britisches Flugangebot auf Basis des Jahres 2015 (Datenquelle: OAG Flugpläne)

\begin{tabular}{|c|c|c|c|c|}
\hline von & nach & Fluggesellschaften & Sitze & Flüge \\
\hline $\mathrm{D}$ & GB & alle & 8.673 .638 & 62.046 \\
\hline GB & D & alle & 8.675 .458 & 62.060 \\
\hline GB & GB & nicht-britische & 2.111 .025 & 22.453 \\
\hline D & D & britische & 32 & 1 \\
\hline \multicolumn{3}{|c|}{ => insgesamt betroffener Luftverkehr } & 19.460 .153 & 146.560 \\
\hline von & nach & Fluggesellschaften & Sitze & Flüge \\
\hline $\mathrm{D}$ & GB & $\begin{array}{l}\text { nicht-britische, nicht deutsche ( } 7 . \\
\text { Freiheit) }\end{array}$ & 1.338 .545 & 7.021 \\
\hline GB & D & $\begin{array}{l}\text { nicht-britische, nicht deutsche ( } 7 . \\
\text { Freiheit) }\end{array}$ & 1.340 .791 & 7.031 \\
\hline GB & GB & deutsche (9. Feiheit) & 30.500 & 610 \\
\hline D & D & britische (9. Freiheit) & 32 & 1 \\
\hline \multicolumn{3}{|c|}{ => gefährdeter Luftverkehr } & 2.709 .868 & 14.663 \\
\hline
\end{tabular}

Welche Fluggesellschaften ihre Strategie überdenken müssten 
Ein Verlust der EU-Mitgliedschaft und der damit einhergehenden Freiheiten der Luft beträfe folglich - und in unterschiedlichem Maße - Fluggesellschaften mit den nachfolgend gelisteten Eigenschaften.

1. Fluggesellschaften, die im Vereinigten Königreich ansässig sind und

a. Verbindungen zwischen dem Vereinigten Königreich und der EU anbieten (vermutlich unkritisch, da 3. und 4. Freiheit)

b. innerhalb der übrigen EU fliegen (7. und 9. Freiheit, Wegfall wahrscheinlich.)

2. Fluggesellschaften aus der EU27 (ohne das Vereinigte Königreich), die

a. Flüge zwischen ihrem Heimatstaat und dem Vereinigten Königreich anbieten (vermutlich unkritisch, da 3. und 4. Freiheit)

b. Flüge zwischen dem Vereinigten Königreich und einem anderen EU-Land als ihrem Heimatland anbieten (7. Freiheit, grds. gefährdet, aber ggf. durch horizontale Klausel in Luftverkehrsabkommen zwischen der EU und GB gesichert)

c. Flüge innerhalb des Vereinigten Königreichs anbieten (9. Freiheit, Wegfall wahrscheinlich)

Fall 1.a. Britische Fluggesellschaften mit starkem Angebot von Großbritannien ins übrige Europa (3. und 4. Freiheit)

Britische Fluggesellschaften, die viele Flüge von Großbritannien in die EU anbieten, sind easyJet und British Airways (Fall 1.a). Für easyJet hatte mehr als jeder zweite Start in Großbritannien (57\%) sein Flugziel in einem anderen EU-Land. Für British Airways war der Anteil ähnlich hoch (44\%). Kleinere Unternehmen kommen zusammen etwa auf einen Marktanteil von einem Drittel, sind aber noch stärker auf Flüge in die EU fokussiert. Der Low Cost Carrier Jet2 etwa flog rund 93\% seiner Passagiere aus Großbritannien in andere EU-Länder, für Monarch Airlines waren es $82 \%$ und für Thomson Airways $71 \%$. Eine Gefährdung dieser Verkehre ist aus den genannten Gründen nicht abzusehen, nicht zuletzt weil britische Urlauber gerade in südeuropäischen Ländern auch eine wesentliche Einnahmequelle darstellen.

Fall 1.b. Britische Fluggesellschaften mit starkem Europaverkehr außerhalb Großbritanniens (7. und 9. Freiheit)

Die führende britische Fluggesellschaft, die mehr als alle anderen die Rechte der 7. und 9. Freiheit im europäischen Luftraum nutzt, etwa für Flüge gänzlich außerhalb Großbritanniens von einem EU-Land zu einem anderen, ist easyJet. In 2015 bot easyJet 22,4 Mio. Sitze auf diesen Verbindungen an und flog dort 1,5 mal so häufig wie im 3.- und 4. Freiheitsverkehr von und nach Großbritannien. Für die Fluggesellschaft British Midland beträgt der Faktor 1,2. Allerdings ist das Angebot von British Midland mit 0,2 Mio. Sitzen im Verkehr zwischen EU-Drittländern relativ klein. Bei einem Verlust der 7.- und 9.-Freiheitsrechte könnten weite Teile des Angebots von easyJet und British Midland nicht aufrechterhalten werden. Es ist daher denkbar, dass diese Fluggesellschaften trotz zusätzlicher administrativer Kosten die Verlegung eines Betriebsteiles in ein EU Land erwägen würden, um so den Zugang zum EU-Binnenmarkt weiterhin zu sichern. Mit easyJet Switzerland verfügt easyJet beispielsweise aus ähnlichen Gründen bereits über Erfahrung mit solchen Ausgründungen.

Fall 2.a. Europäische (nicht-britische) Fluggesellschaften mit starkem Angebot zwischen Heimatstaat und Großbritannien (3. und 4. Freiheit) 
Fluggesellschaften aus EU-Ländern, die viele Flüge von dort in das Vereinigte Königreich anbieten, wären, wie die britischen Fluggesellschaften im Fall 1.a, ebenfalls kaum von einem Brexit betroffen. Dies betrifft in erster Linie die europäischen Hub-Carrier und Low Cost Carrier wie Ryanair, Aer Lingus oder Eurowings. Für Lufthansa machten Großbritannienverkehre im Jahr 2015 beispielsweise 8\% ihres innereuropäischen Sitzplatzangebots aus, für die Tochter Eurowings/Germanwings waren es 14\%. Bei Air France haben Großbritannienverkehre mit der Heimat einen Anteil von 6\% am innereuropäischen Verkehr der Airline und bei Ryanair 7\%. Anders sieht es bei Aer Lingus aus. Die ehemalige irische National- Fluggesellschaft bietet $51 \%$ ihres Europaverkehrs zwischen Großbritannien und der Heimat an.

\section{Fall 2.b. Europäische (nicht-britische) Fluggesellschaften mit starkem Angebot zwischen Großbritannien und einem fremden EU-Land (7. Freiheit)}

Fluggesellschaften, die in einem europäischen Land (außer Großbritannien) beheimatet sind, aber viel zwischen Großbritannien und „fremden“ EU-Ländern fliegen, müssen theoretisch mit einem Verlust der hierfür benötigten Rechte der 7. Freiheit rechnen. Für Ryanair basieren etwa 30 Mio. angebotene Sitze auf diesen Rechten, also ein Drittel (29\%) ihres Gesamtangebots. Allerdings kämen auch einige kleinere Fluggesellschaften in Schwierigkeiten. Beim Low Cost Carrier Wizz Air beispielsweise wäre ebenfalls fast ein Drittel (28\%) seiner Flüge betroffen, und bei der irischen CityJet, die hauptsächlich von London-City aus in europäische Metropolen fliegt, sogar $71 \%$. Damit wäre das derzeitige Geschäft dieser Fluggesellschaften prinzipiell stark durch einen Brexit gefährdet. In der Praxis ist jedoch zumindest mittelfristig, etwa nach bilateralen Neuverhandlungen, die vom EUGH geforderten horizontale Klauseln gelten müssten, womit Verkehre der 7. Freiheit von EU-Airlines nach Großbritannien erlaubt blieben bzw. wieder erlaubt werden müssten.

Fall 2.c. Europäische (nicht-britische) Fluggesellschaften mit starkem Angebot innerhalb von Großbritannien (9. Freiheit)

Fluggesellschaften, die in einem europäischen Land (außer Großbritannien) beheimatet sind, aber innerhalb Großbritanniens operieren, sind in erster Linie ebenfalls Ryanair und Aer Lingus. Es ist nicht damit zu rechnen, dass bei einem Brexit die hierfür nötigen Kabotagerechte (9. Freiheit) erhalten blieben. Allerdings spielt dieses Marktsegment im innereuropäischen Verkehr insgesamt prozentual kaum eine Rolle (0\%) und macht selbst bei Ryanair lediglich $1 \%$ der innereuropäischen Flüge aus, bei Aer Lingus 5\%.

\section{Konsequenzen}

Fluggesellschaften mit einem hohen Verkehrsanteil in den Kategorien 1.b., 2.c. und ggf. 2.b. würden durch einen Wegfall der bisher genutzten Freiheiten der Luft gezwungen sein, sich strategisch neu zu orientieren. Dies könnte etwa die Neugründung von Tochtergesellschaften im EU-Raum (für britische Fluggesellschaften) oder in Großbritannien (für EU-Fluggesellschaften) bedeuten, oder eine Verlagerung der Flotte auf andere Strecken im europäischen Binnenmarkt, was in der Folge auch die Wettbewerbssituation innerhalb Europas verändern könnte.

\section{Auswirkung auf die Luftverkehrsnachfrage}


Selbst wenn man die strategischen Umstrukturierungen von Fluggesellschaften nach einem möglichen Brexit außen vor lässt, ist allein aufgrund der veränderten wirtschaftlichen Situation mit nachfrageinduzierten Veränderungen des Luftverkehrsangebots zu rechnen.

Durch einen Brexit, so prognostiziert der IWF, ginge im Vereinigten Königreich die wirtschaftliche Aktivität zurück, was zu Einkommensverlusten (Arbeits- und Kapitaleinkommen) führen würde. ${ }^{19}$ Großbritannien und Nordirland bilden mit einem Anteil von 17,6\% am BIP im Jahr 2015 die zweitgrößte Volkswirtschaft der EU. Insofern würde sich eine Abschwächung der wirtschaftlichen Dynamik aufgrund eines Brexits auch auf die Passagierströme etwa zwischen Deutschland und Großbritannien auswirken.

Es stellt sich die Frage, wie stark die wirtschaftliche Dynamik im Vereinigten Königreich aufgrund eines Brexits abnehmen würde. Hierfür liegen von verschiedenen Instituten Szenarien vor, wobei die beiden Szenarien des IWF in etwa die beiden Extrempunkte der verschiedenen Studien widergeben. ${ }^{20}$ In einem ersten Szenario sinkt das BIP-Wachstum um 0,8\%-Punkte in 2017 und 0,4\%-Punkte in 2018 gegenüber dem Basisszenario einer EU-Mitgliedschaft ab. Hierbei wird angenommen, dass Großbritannien gegenüber der EU einen Status ähnlich wie Norwegen oder die Schweiz erhält. Im zweiten Szenario des IWF schrumpft das BIP-Wachstum um 3,0\%-Punkte in 2017 und 1,6\%-Punkte in 2018 gegenüber dem Status Quo. Es wird dabei unterstellt, dass die Beziehungen zwischen zur EU lediglich nach den Standards der WTO geregelt werden.

Auf Basis der BIP-Schätzungen des IWF wird im Folgenden in drei Nachfrageszenarien die Luftverkehrsentwicklung an deutschen Flughäfen prognostiziert. Das Prognosemodell basiert auf einer multiplen Regressionsanalyse, welche die jährlichen Wachstumsraten in Abhängigkeit von erklärenden Größen prognostiziert. ${ }^{21}$ Dies sind im Kern das Wirtschaftswachstum (BIP), das Flugzeuggrößenwachstum und außerordentliche positive oder negative Einflüsse, wie zum Beispiel 9/11.22

1. Nachfrageszenario "Status Quo": Kein Brexit

2. Nachfrageszenario "EFTA-Status": Vollzug des Brexits; Großbritannien erhält gegenüber der EU ein Status ähnlich wie die EFTA-Staaten Norwegen und Schweiz

3. Nachfragesenario "WTO-Status": Vollzug des Brexits; Regelung der Beziehungen zwischen Großbritannien und der EU lediglich nach den Standards der WTO

Szenario "Status Quo"

Im Status Quo, d.h. ohne Brexit, prognostizieren wir für das Jahr 2017 ein Passagierwachstum an deutschen Flughäfen von 2,9\% und für das Jahr 2018 einen Wert von 2,5\%. Entsprechend erwarten wir unter diesen Bedingungen ein Flugbewegungswachstum von 1,4\% für 2017 und $1,3 \%$ für 2018.

\footnotetext{
${ }^{19}$ Vgl. Internationaler Währungsfonds (IWF): IMF Country Report No. 16/169, Washington D.C. 2016.

$20 \mathrm{Vgl}$. ebd.

${ }^{21}$ Zur Prognosemethodik vgl. z.B.: M.C. Gelhausen, P. Berster, D. Wilken: A New Model of Long Term Forecasting Air Passenger Demand and the Number of Air Transport Movements of Germany, in: Proceedings of ATRS World Conference, Rhodes 2016.

${ }^{22}$ Als Prognose für das BIP der EU dient die Datenbank des World Economic Outlook 2016 (Stand Juni 2016) des Internationalen Währungsfonds (2016). Das prognostizierte Flugzeuggrößenwachstum beträgt $1,5 \%$ in 2017 und 1,2\% in 2018.
} 
Tabelle 3: Prognoseergebnisse für deutsche Flughäfen im Szenario „Status Quo"

\begin{tabular}{lcc}
\hline & $\mathbf{2 0 1 7}$ & $\mathbf{2 0 1 8}$ \\
\hline Passagiere & $2,87 \%$ & $2,52 \%$ \\
Flugbewegungen & $1,41 \%$ & $1,33 \%$ \\
\hline
\end{tabular}

Szenarios "EFTA-Status" und "WTO-Status"

Gegenüber dem Status Quo würden die Wachstumsraten von Passagieren und Flugbewegungen im EFTA-Szenario jeweils um etwa 0,1\%-Punkte niedriger liegen. Im WTO-Szenario würden die Wachstumsraten sogar um etwa 0,3\%-Punkte in 2017 und 0,2\%-Punkte in 2018 gegenüber dem Status Quo fallen. Tabelle 4 zeigt zusammengefasst die Prognoseergebnisse unter Berücksichtigung eines Brexits unter den Annahmen des IWF bezüglich der wirtschaftlichen Entwicklung in Großbritannien. Hierbei ist zu beachten, dass die wirtschaftlichen Auswirkungen eines Brexits hauptsächlich in 2018 und später greifen werden und dass

Anpassungsmaßnahmen die Folgen noch dämpfen könnten. Andererseits kann die nun anhaltende Planungsunsicherheit zu einem (teilweise) vorzeitigen Eintreffen der Effekte führen.

Zum Vergleich: Die IATA erwartet eine Dämpfung des Passagierwachstums in Großbritannien und Nordirland von etwa 1 bis 1,5\%-Punkte pro Jahr. ${ }^{23}$ Würde man über die Verkehrsverflechtungen diesen Wert mit dem Verkehrsanteil Deutschland - Großbritannien multiplizieren, so ergäbe sich eine ungefähre Orientierungsgröße von 0,1 bis 0,15\%-Punkten, um die Passagiere und Flugbewegungen an deutschen Flughäfen aufgrund eines Brexits weniger wachsen würden.

Insofern halten wir bei unserer Prognose das EFTA-Szenario, d.h. eine Dämpfung des Passagierund Flugbewegungswachstum um jeweils etwa 0,1\%-Punkte gegenüber dem Status Quo, für das wahrscheinlichste.

Tabelle 4: Prognoseergebnisse für deutsche Flughäfen in den Szenarien „EFTA" und „WTO”

\begin{tabular}{lcccc}
\hline & \multicolumn{2}{c}{ EFTA-Szenario } & \multicolumn{2}{c}{ WTO-Szenario } \\
& $\mathbf{2 0 1 7}$ & $\mathbf{2 0 1 8}$ & $\mathbf{2 0 1 7}$ & $\mathbf{2 0 1 8}$ \\
\hline Passagiere & $2,76 \%$ & $2,47 \%$ & $2,45 \%$ & $2,30 \%$ \\
Flugbewegungen & $1,30 \%$ & $1,28 \%$ & $0,99 \%$ & $1,11 \%$ \\
\hline
\end{tabular}

\section{Wechselkurseffekte}

Basierend auf den Analysen der IATA ${ }^{24}$ und HM Treasury ${ }^{25}$ wirken sich die zu erwartenden Wechselkursänderungen zwischen dem britischen Pfund und dem Euro aufgrund der Verkehrsstruktur zwischen Deutschland und Großbritannien positiv aus. Das Verhältnis zwischen

\footnotetext{
${ }^{23} \mathrm{Vgl}$. International Air Transport Association (IATA): The Impact of Brexit on UK Air Transport, Montreal 2016.

24 Vgl. IATA, a.a.O.

${ }^{25} \mathrm{Vgl}$. HM Government: HM Treasury Analysis: The Immediate Economic Impact of Leaving the EU (Cm9292), London Mai 2016.
} 
Quell- und Zielverkehr zwischen deutschen und britischen Flughäfen liegt bei 55\% zu 45\% bzw. im reinen Privatreiseverkehr bei $62 \%$ zu $38 \%$, so dass insgesamt mehr von Deutschland nach Großbritannien geflogen wird als umgekehrt. Insofern würde eine Abschwächung des britischen Pfundes gegenüber dem Euro die Kaufkraft von Bürgern der Eurozone in der Pfundzone stärken und im Gegenzug die Kaufkraft von Bürgern der Pfundzone in der Eurozone schwächen.

Aufgrund des verhältnismäßig starken Quellverkehrs würde der Nettoeffekt aus deutscher Sicht positiv sein, wie Tabelle 5 zeigt. Hierbei liegen eine Abwertung des Pfundes von 12\% sowie eine BIP-Elastizität von 1,34 zugrunde. Zwei Szenarien werden unterschieden: Im ersten Szenario reagiert der gesamte Passagiermarkt, d.h. Geschäfts- und Privatreisende, auf die Wechselkursänderungen. Im zweiten Fall reagieren nur die Privatreisenden, die ca. 60\% des Gesamtmarktes darstellen. Der Nettoeffekt beträgt in beiden Fällen etwa 0,2\%.

Tabelle 5: Effekte möglicher Wechselkursänderungen basierend auf Annahmen von HM Treasury

\begin{tabular}{lccc}
\hline & Quellverkehr & Zielverkehr & Nettoeffekt \\
\hline Privat \& Geschäft & $0,92 \%$ & $-0,76 \%$ & $0,16 \%$ \\
Privat & $0,62 \%$ & $-0,38 \%$ & $0,24 \%$ \\
\hline
\end{tabular}

Insofern könnte durch den Wechselkurseffekt (Einmaleffekt) der oben dargestellte Nachfragerückgang unter Umständen zumindest teilweise ausgeglichen werden. Allerdings sind diese Effekte mit großer Unsicherheit behaftet und basieren auf den Wechselkursannahmen von HM Treasury. Insgesamt ist aus unserer Sicht im EFTA-Szenario aufgrund der beiden gegenläufigen Effekte eher mit keiner signifikanten Nettonachfrageänderung durch einen möglichen Brexit zurechnen, so dass die Prognoseergebnisse des Status Quo Anwendung finden. Für das WTO-Szenario könnte die Nettonachfrageänderung insgesamt in einem Bereich von etwa 0,3\%-Punkten liegen, um die die Ergebnisse des Status Quo reduziert werden müssten.

\section{Fazit}

Rund ein Viertel des derzeitigen Flugangebots in Europa betrifft in irgendeiner Form das Vereinigte Königreich, das für Deutschland das weltweit zweithäufigste ausländische Anflugziel darstellt. Selbst im tatsächlichen Falle eines Brexits wäre damit zu rechnen, dass ein großer Teil dieser Flüge angebotsseitig nicht betroffen wäre, da das Vereinigte Königreich nach dem Austritt aus der EU neue Luftverkehrsabkommen zumindest auf bilateraler Ebene für den zwischenstaatlichen (3.- und 4.-Freiheits-) Verkehr verhandeln würde oder alte bilaterale Verträge wieder auflebten.

Es ist indes deutlich wahrscheinlicher, dass Verkehre der 7.-9. Freiheit nach einem Brexit nicht mehr möglich wären. Das beträfe zum einen Flüge britischer Fluggesellschaften innerhalb oder zwischen anderen EU-Ländern, zum anderen Flüge nicht-britischer Fluggesellschaften innerhalb Großbritanniens oder ggf. auch zwischen Großbritannien und einem EU-Land, das nicht ihr Heimatland ist. Im Jahr 2015 wurden für 14\% der Flüge und 16\% der Sitze diese 7.-9. Freiheitrechte im innereuropäischen Luftverkehr genutzt. Ihr Wegfall würde einige europäische Fluggesellschaften zu strategischen Neuausrichtungen zwingen. Besonders betroffen wären die 
großen Anbieter easyJet und ggf. Ryanair, aber auch spezialisiertere Firmen wie British Midland, Wizz Air oder CityJet, während sich für Lufthansa, Air France oder British Airways, die vornehmlich im bilateralen 3.- und 4.-Freiheitsverkehr tätig sind, nicht viel ändern würde. ${ }^{26}$ Für den deutschen Luftverkehr ergibt sich eine prinzipielle Gefährdung von „nur" 2,7 Mio. Sitzen auf weniger als 15.000 Flügen, die beispielsweise von Ryanair nach Großbritannien oder von easyJet in andere EU-Staaten durchgeführt werden.

Standortentscheidungen und Netzveränderungen von Fluggesellschaften hätten direkte Auswirkungen auf das Verkehrsaufkommen an Flughäfen somit wirtschaftliche Effekte für die Flughafenregionen. Spekulationen gibt es viele. So könnte die Bedeutung von London als Drehkreuz schwinden und beispielsweise Frankfurt davon profitieren. Auch könnten Low Cost Carrier wie Ryanair, die Kapazitäten aus dem Großbritannienverkehr abziehen müssten oder wollten, mit diesen ihre Präsenz im übrigen Europa verstärken. Eine solche Marktstrukturänderung könnte die Wettbewerbssituation in Europa deutlich verschärfen. Während sich Verbraucher über niedrigere Preise freuen könnten, wäre es möglich, dass etablierte Fluggesellschaften weiter unter Druck gerieten. Ryanair CEO O'Leary hat betont, in Deutschland größter Anbieter nach Lufthansa werden zu wollen. ${ }^{27}$ Somit könnten Lufthansa und airberlin, die direkt kaum vom Brexit betroffen sind, dennoch unter Druck geraten.

Der IWF und andere Institute rechnen mit einem Rückgang der Wirtschaftskraft Großbritanniens nach einem Brexit. Auf dieser Basis prognostizieren wir für Deutschland unabhängig von eventuellen angebotsseitigen Umstrukturierungen die Veränderung des Luftverkehrsaufkommens in verschiedenen Szenarien. Ohne Brexit, also im "Status Quo", würde das Passagieraufkommen an deutschen Flughäfen in 2017 um 2,9\% und in 2018 um 2,5\% steigen. Bei einem "WTO-Szenario", das heißt die Beziehungen zwischen dem Vereinigten Königreich und der EU würden lediglich nach den Standards der WTO geregelt, betrüge die Dämpfung 0,2\%-Punkte in 2017, und 0,3\%-Punkte in 2018. Im realistischeren EFTA-Szenario, in dem das Vereinigte Königreich nach dem EU-Austritt einen ähnlichen Status wie Norwegen oder die Schweiz bekäme, würde in 2017 und 2018 eine Dämpfung des Passagier- und Flugbewegungswachstum um 0,1\%-Punkte gegenüber dem Status Quo eintreten. Währungseffekte könnten diesen Rückgang eventuell zumindest zum Teil noch auffangen.

Die Folgen eines Brexits für den Luftverkehr in Deutschland könnten somit auf der Angebotsseite durch Marktumstrukturierungen deutlich heftiger ausfallen als es die nachfrageorientieren Prognosen vermuten lassen.

\footnotetext{
${ }^{26}$ Etwaige Probleme für British Airways aufgrund der britisch-spanischen Holdingstruktur sind hier nicht berücksichtigt.

${ }^{27}$ Vgl. Die Zeit: Eurowings ist zum Scheitern verurteilt, Interview mit Michael O'Leary vom 9.6.2016, http://www.zeit.de/2016/23/ryanair-chef-michael-o-leary-airlines (14.7.2016).
} 